\title{
The Ruesch Center Symposium 2014: Fighting a Smarter War against Cancer Using Immunotherapy
}

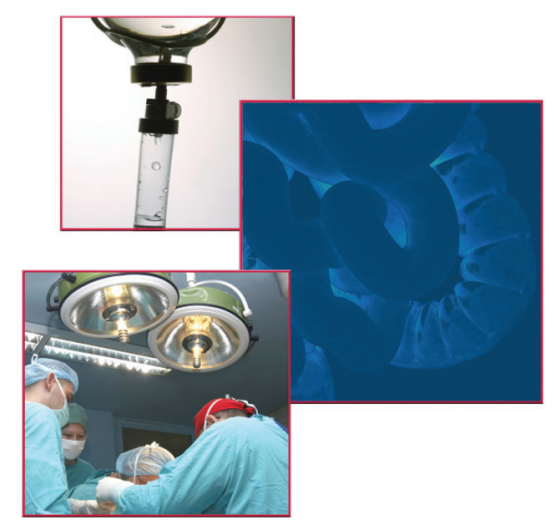

\author{
Marion L Hartley ${ }^{*}{ }^{1}$ \& John L Marshall ${ }^{1}$ \\ The Ruesch Cancer Symposium, Washington DC, USA, 4-6 December 2014 \\ Keywords: checkpoint inhibitor $\bullet$ Gl cancers $\bullet$ immunotherapy $\bullet$ Ruesch Symposium $\bullet$ \\ vaccine
}

\section{The symposium}

Every year the Ruesch Center runs an annual symposium: Fighting a Smarter War against Cancer. Although not on the scale of the American Society of Clinical Oncology (ASCO) meetings, the symposium is overbrimming with oncology expertise. The event convenes not only national but also global experts and always broaches the latest hot or oftentimes controversial topics [1].

This year the center's annual symposium (2014) had a major focus on immune therapy for GI cancers and on Friday, December 5, we brought together 'an incredible line-up' of immunotherapy experts (in speaker Chris Heery's words). Running themes throughout the symposium were that "we are entering a new era in the treatment of GI cancer", "we are beginning to see a transformation in the standard of care", "immunotherapy is the new treatment Paradigm" and "there has been a fundamental shift in the way we understand cancer".

As a lead-up to Friday, the session on Thursday evening discussed the latest advances in GI cancer research, during which Michael Pishvaian likened surgery to uprooting the large weed growing in the middle of the garden while chemotherapy was like treating the whole yard for unwanted growth. On Friday it was made clear that immunotherapy is capable of doing both these things and as such is a tactical gamechanger. Saturday was a day for patient education, covering a range of topics aimed at increasing patient knowledge of cancer research, therapy and coping skills. This commentary will cover the events on Thursday and Friday only.

\section{Symposium presenters}

Thursday: Advances in Gl cancer 2014

Michael Pishvaian, MD, PhD; Brandon Smaglo, MD; John Marshall, MD; Patrick Jackson, MD; Waddah B Al-Refaie, MD, FACS; Lynt Johnson, MD, MBA; Lana Bijelic, MD; Thomas Stahl MD; Ruth He, MD, PhD; Mohamed Salem, MD; James Shaw, MD, MPH; Raymond Wadlow, MD; Alexander Kim, MD; Keith Unger, MD.

Friday: Immunotherapy for GI cancers John Marshall, MD; Jeff Schlom, PhD; Tim Greten, MD; Bernard A Fox, PhD (keynote speaker, Schafer Lecture); Chris Heery, MD; Neil Segal, MD, PhD; Michael Morse, MD, FACP, MHS; Elizabeth Jaffee, MD.

Speakers' slides can be viewed on the Ruesch Center, Georgetown University Website [2].

\footnotetext{
${ }^{1}$ The Ruesch Center for the Cure of GI Cancers, Lombardi Comprehensive Cancer Center, Georgetown University, 3800 Reservoir Road, NW 20007, USA

*Author for correspondence: mlh95@georgetown.edu
} 
John Marshall, Director of the Ruesch Center, kicked off Thursday's session with opening remarks, during which he put forward that people are generally reserved in the use of the word 'cure' as it relates to cancer but cures are exactly what the research community should strive for.

Partnerships and collaborations are key and the effectuation of large scale - even global clinical trials encompassing state of the art treatments are central to finding those cures. Indeed, many symposium participants have worked and are working in collaboration with each other on cutting edge immunotherapy research in the field of GI cancer. Immunotherapy is the current preoccupation; everyone believes that this approach is driving the new cancer therapy era. However, GI cancers are still ultimately being left at the sidelines and the mutual aim of symposium attendees is to bring development of life-saving GI cancer therapies up to a level that is in line with the 'hot topic' cancers such as breast and melanoma. Increased GI cancer funding is central to this accomplishment.

Raymond Wadlow, moderator of Thursday's sessions, emphasized that patients are our most precious resource and that we need to direct their participation in the most fruitful way possible. This sentiment continued throughout the symposium: speakers repeatedly thanked patients for their participation in clinical research, for their graciousness regarding provision of blood and tissue biopsies, and their time - for being heroes. All Thursday presenters spoke of progress made in the treatment of GI cancers over the last few years, endorsing the use of individualized therapy and molecular profiling and reiterating that there is 'new hope on the horizon,' particularly with the emergence of immunotherapy. We have gone as far as we can with standard of care (SOC) and it is time to move the bar forward. Immunotherapy is a form of personalized medicine because tumor cell antigen release and subsequent immune response can be unique to an individual and their tumor. With immune therapy we are not directly treating the tumor but manipulating the patient's immune system.

The verity of two common beliefs was to be clarified during symposium discussions:

(1) People develop cancer due to their having a defective or faulty immune system;

(2) Cancer is 'self'. Therefore there is no hope.

These will be revisited at the end of this commentary.

\section{Immunotherapy}

Background knowledge gained during

symposium discussions

Chemotherapy can affect the growth rate of tumors only while it is being administered. Moreover, toxicity or resistance often results in early cessation of therapy.

'Immune' therapy (immunotherapy) on the other hand has an indirect effect on the tumor and this effect is usually delayed as the immune system is manipulated into action and does its work. Therefore, doctors and patients alike must be patient; substantial improvements in overall survival are usually observed with the correct immune treatment and, theoretically, there is a correct treatment for everyone. Bernard Fox, who is specializing in immune therapy for colorectal cancer, said that we now have ways to turn the immune system on and keep it on. Immune treatment regimens can continue for long periods of time and there are few adverse effects and no tumor resistance.

A good deal of clinical research has shown that overall survival is a much more accurate marker of immunotherapy success than is TTP (time to progression) - classically used to judge chemotherapy effects $[3,4]$.

The first step in the tumor immunity process involves the release of tumor cell antigens, which are bound by antigen presenting cells, predominantly dendritic cells. This process subsequently leads to priming and activation of T cells. Activated $\mathrm{T}$ cells (effector $\mathrm{T}$ cells $\left[\mathrm{T}_{\text {effs }}\right]$ ) migrate to and infiltrate the tumor, subsequently killing the malignant cells [5]. Researchers and pharmaceutical companies are currently developing therapies to intervene at every stage of this process and a few of these were presented at the symposium.

If patient tumors are not already immunogenic (displaying an active $\mathrm{T}$ cell response to tumor cells), destructive therapies such as chemotherapy, radiation therapy and targeted therapy can promote immunogenicity, as can treatment with vaccines [6].

The number and type of tumor-infiltrating $\mathrm{T}$ cells can now be assessed from a patient tumor biopsy [7]. Tumor infiltrate is actually highly prognostic of patient outcome (shown for patients with colorectal cancer [7]) - much more so than TNM staging [8]. An 'Immunoscore' is calculated by measuring $\mathrm{T}_{\text {effs }}$ that infiltrate the tumor - the more there are, the stronger the immune response against the tumor. The score is 
based on the enumeration of two $\mathrm{T}_{\text {eff }}$ populations (CD3 to CD45R0, CD3 to CD8 and CD8 to $\mathrm{CD} 45 \mathrm{R} 0$ ) in the core and also in the invasive margins of the tumor.

However, we cannot preoccupy ourselves only with $\mathrm{T}_{\text {effs }}$. Regulatory $\mathrm{T}$ cells $\left(\mathrm{T}_{\text {regs }}\right)$ also exist that are responsible for putting the brakes on an immune response - these inhibitory signals prevent autoimmunity in normal cells but are bad news in the war against cancer. $\mathrm{T}_{\text {regs }}$ can also be measured in tumor infiltrate and cancer researchers are aiming to find a way to increase $\mathrm{T}_{\text {effs }}$ while decreasing $\mathrm{T}_{\text {regs }}-$ it is an intricate balance.

Molecules such as CTLA-4 and PD-1 (induced on T cells) and PD-L1 (induced on the tumor) regulate $\mathrm{T}$ cell response after the immune response has been activated and are thus termed immune checkpoints. Monoclonal antibodies called checkpoint inhibitors have been found to reduce or eliminate these negative/inhibitory signals and effect a significant antitumor response, first seen in melanoma [9] and now being assessed in GI cancers. These are very promising results but it should be noted that checkpoint inhibitors will be effective only if a tumor first elicits a $T$ cell response.

\section{Gl cancer-related research}

Symposium presenters addressed the problems currently facing immunotherapy research. Some of these are listed below:

- As a regulatory requirement, cancer treatments are initially studied preclinically in human tumor xenograft mouse models. In the case of immune therapies, this is good initial practice regarding toxicity but rather a pointless exercise scientifically: to implant a human tumor into a mouse, the mouse must first be immunosuppressed, which defeats the object of immune study entirely, and the alternative study of mouse tumors in mice has no relevance at all to the human scenario. Therefore, it makes sense that science be studied in patient clinical trials. This makes for an accurate but potentially lengthy and expensive exercise. Therefore, more funding is needed and trials need to be efficient so that we can find answers in a timely fashion. Methods of patient selection are key to the creation of these efficient, essentially small-scale, trials. Chris Heery proposed that a $\mathrm{T}$ cell-type analysis in tumor infiltrate should be used for GI cancer patient selection. For example, the presence of a high number of $\mathrm{T}_{\text {effs }}$ would tell us that a patient does not need to undergo cytotoxic $T$ lymphocyte activation, while the presence of Teffs would tell us that checkpoint inhibitors are a valid option for study;

- Currently accepted clinical trial design requires a SOC arm for comparative purposes and this regulatory necessity is leading to increasing problems with clinical trial accrual and completion: patients read and understand that something better than SOC is out there, so why would they enter a trial to end up on the SOC arm? The thing is that until more is understood about tumor immunology and how to exploit it, immunotherapy is not necessarily the panacea many have been led to believe;

- Because SOC is approved for first- and second-line treatment in most cancers, immunotherapy is not administered until patients are pretty beaten up from several lines of chemotherapy. This is not ideal because immunotherapy seems to be more effective the earlier it is given.

Bernard Fox gave our Schafer Memorial Lecture [10] for the year and began with immunotherapy in the National Press: The Wall Street Journal recently reported how immune therapy is transforming oncology [11], while Science magazine heralded immunotherapy, particularly checkpoint inhibitors, the breakthrough of the year 2013 [12] and Oncology Live headlined: New Paradigms Emerge for Translating Immunotherapy Into Broad Clinical Use [13]. The authors noted that we are starting to see results with immunotherapy in solid tumors including GI cancers. Again, despite all the hype and some fantastic results, researchers still do not know how to make immunotherapy work for everyone. The general public should not be misled.

The Society for Immunotherapy of Cancer is funding a study by Fox that includes 17 countries and 23 sites in a retrospective analysis involving 5-6000 patients with colorectal cancer. The study aims to validate the immunoscore as the new prognostic biomarker and modify the use of TNM classification.

Fox hypothesized that there is a positive correlation between the number of known mutations in a tumor type and immunogenicity. Colorectal cancer has quite a high number of mutations [14] 
and tumor immunogenicity would be expected in patients with this disease (confirmed by a positive immunoscore). A recent paper published in Nature [15] indicated that almost 50\% of colorectal tumors tested positive for PD-L1. Thus there is reason to believe that immunoscore-positive colorectal cancer patients will respond well to PD-L1 inhibition.

Fox's group has also developed a tumorderived autophagosome-enriched vaccine called DPV-001 [16]. This vaccine contains a number of NCI prioritized cancer antigens, along with single nucleotide gene variations that are shared with colorectal tumors from patients.

Many types of cancer treatment vaccines have been studied [17]. Generally, these vaccines introduce antigens into the body (sometimes one; sometimes more), which basically teach dendritic cells to process antigens and then bind to $T$ cell receptors, usually facilitated by co-stimulation (co-stimulatory molecules contained within the vaccines). T cells become activated and then put up clonal $\mathrm{T}$ cell receptors against that antigen. Thus immunogenicity begins.

Jeff Schlom has dedicated his career to cancer immunology and immunotherapy [18], particularly vaccines. More recently he and his collaborators at the Laboratory of Tumor Immunology and Biology have been focusing on recombinant yeast-brachyury as a potential vaccine in cancer therapy [19]. The rationale behind a brachyuryrelated vaccine is that when brachyury (an embryologic transcription factor) is overexpressed in epithelial cell cancers, such as colon cancer, epithelial mesenchymal transition is induced, causing cells to become fibroblastoid-like, migratory and invasive (processes involved in metastasis). It is very new to be able to target a metastatic process and Heery is running two trials at the $\mathrm{NCI}$, as well as one trial with Marshall, using brachyury vaccination in colorectal and pancreas cancer patient populations. The vaccine structure has a tumor antigen (brachyury) portion and a costimulatory (TRICOM) portion.

Schlom and Marshall, as a prerequisite for further trials, are collaboratively looking at all immune subsets in patients receiving a FOLFOXbevacizumab combination (SOC) to identify which patients could go on to benefit from immunotherapy. Additionally, an association between frequency of $\mathrm{T}_{\text {regs }}$ post-therapy with FOLFIRI (SOC) and subsequent response to immunotherapy is being assessed. It is predicted that patients with less $\mathrm{T}_{\text {regs }}$ have a better prognosis.

Michael Morse collaborated with John Marshall on the treatment of patients with resected metastatic colorectal cancer with the PANVAC $\mathrm{V} / \mathrm{F}$ vaccine, which combines two tumor antigens, CEA and MUC-1 with TRICOM [3]. They found that treated patients experienced a startling $90 \%$ overall survival increase.

Elizabeth Jaffee's research has focused on immune treatment for pancreas cancer. Her team confirmed that vaccines can induce tumorinfiltrating lymphocytes in traditionally 'nonimmunogenic' tumors and thus pancreatic cancers can be made immunogenic. Jaffee has had much success with GVAX - a dendritic cell recruiting vaccine that contains two genetically modified allogeneic tumor cell lines expressing GM-CSF that provokes activating $\mathrm{T}$ cells. Although she has seen huge $\mathrm{T}$ cell responses to vaccination in some of her patients with pancreatic cancer - and in a few cases an impressive anti-tumor response - Jaffee heeds that T lymphocyte activation is in most cases a sign of immune system modulation, not curative success. There is still much work to be done and a lot to learn. There have been thoughts that two vaccines could be better than one. For example, Jaffee and her team have just completed a PRIME/BOOST study in patients with pancreatic cancer using vaccines targeting two arms of the $\mathrm{T}$ cell response (GVAX and a listeria-based vaccine) together with cyclophosphamide [20]. This combination extended survival while causing minimal toxicity.

It is understood that other immune pathways may intervene in ways we have yet to understand. Jaffee has further found that activated tumor-infiltrating lymphocytes in turn secrete IFN $\gamma$ and other cytokines that upregulate the PD-1/PD-L1 and other immune-modulating pathways. As $\mathrm{T}$ cells seem to get downregulated by suppressive mechanisms within the tumor (which include PD-1/PD-L1, as well as CTLA4 and others), vaccines should probably be given alongside agents that modulate these suppressive mechanisms to fully activate the T cell response. Indeed, in one study, a GVAX-ipilumumab combination had the dramatic effect of complete remission in one patient with metastatic pancreatic cancer. Again, we cannot forget the patients who did not respond in this way - there is still much to elucidate.

Neil Segal has done much research on checkpoint inhibitors and treatment combinations, as 
well as the use of tumor infiltrate to enhance response to this therapy. He has seen profound clinical benefit, albeit in a subset of patients [21]. $\mathrm{He}$ also established that all GI cancers tested (colorectal, gastric, pancreas and hepatocellular) can be recognized by the immune system (are immunogenic).

Tim Greten and his team have been involved with a clinical study, the results of which are yet unpublished, where destruction of hepatocellular carcinoma by ablative therapies such as transarterial catheter chemoembolization (TACE) or radiofrequency ablation (RFA), accompanied by dampening/blocking of immune-suppressive mechanisms with the antiCTLA-4 checkpoint inhibitor, tremelimumab can lead to a large increase in the frequency of tumor infiltrating $\mathrm{CD}^{+}{ }^{+} \mathrm{T}$ cells (ClinicalTrials.gov: NCT01853618).

\section{Summary}

To summarize, we must revisit the 2 common beliefs stated at the beginning of the immunotherapy session:

1. Do people develop GI cancer because of a faulty immune system? The answer lies in the facts laid before us by speakers on Friday: the fault lies generally not in immune system function but in the immunogenicity of tumor antigens and the ability of tumors to evade the immune response by signaling overexpression of certain factors;

2. Is cancer seen as 'self'? Sometimes yes and sometimes no. In either case, there is hope. Research has shown that we can manipulate the immune system to overcome tumor antigen properties and other barriers to the desired immune response.

Immunotherapy has some unique properties:
1. Minimal toxicity (QOL);

2. The effect is on the host immune system so it has an indirect effect on tumor, which may be delayed as the immune system does its work;

3. Overall survival is the appropriate primary endpoint (as opposed to time to progression or RECIST);

4. Induction of host immunity is a dynamic process that can persist postimmunotherapy;

5. There is the potential for an enhanced effect on concomitant or subsequent therapies.

We still need to learn more about how signals interact and progress. Understanding the underlying biology should lead to a better use of agents and better trial design. Therapies discussed in this review focus on immune response induction, inhibition of immune checkpoints and combination with standard agents. Other types of immune modulators not discussed here are undergoing study and should help to fit the pieces of the immunotherapy puzzle together. Our future direction should incorporate well planned and efficient immunotherapy trials as well as further study of biomarkers of immune response for use as predictors of efficacy and markers of therapeutic success.

\section{Financial \& competing interests disclosure}

The authors have no relevant affiliations or financial involvement with any organization or entity with a financial interest in or financial conflict with the subject matter or materials discussed in the manuscript. This includes employment, consultancies, honoraria, stock ownership or options, expert testimony, grants or patents received or pending, or royalties.

No writing assistance was utilized in the production of this manuscript.

\section{References}

1 Marshall J. Conference scene: fighting a smarter war against cancer. Colorectal Cancer 3, 131-133 (2014).

2 Georgetown Lombardi Comprehensive Cancer Center. https://ruesch.georgetown.edu

3 Morse MA, Niedzwiecki D, Marshall JL et al. A randomized Phase II study of immunization with dendritic cells modified with poxvectors encoding CEA and MUC1 compared with the same poxvectors plus GM-CSF for resected metastatic colorectal cancer. Ann. Surg. 258, 879-886 (2013).
4 Schlom J. Therapeutic cancer vaccines: current status and moving forward. J. Natl Cancer Inst. 104, 599-613 (2012).

5 Chen DS, Mellman I. Oncology meets immunology: the cancer-immunity cycle. Immunity 39, 1-10 (2013).

6 Chen G, Gupta R, Petrik S et al. A feasibility study of cyclophosphamide, trastuzumab, and an allogeneic GM-CSF-secreting breast tumor vaccine for HER2+ metastatic breast cancer. Cancer Immunol. Res. 2, 949-961 (2014). 
7 Galon J, Costes A, Sanchez-Cabo F et al. Type, density, and location of immune cells within human colorectal tumors predict clinical outcome. Science 313, 1960-1964 (2006).

8 Galon J, Mlecnik B, Bindea G et al. Towards the introduction of the 'Immunoscore' in the classification of malignant tumours. J. Pathol. 232, 199-209 (2014).

9 Ribas A, Hersey P, Middleton MR et al. New challenges in endpoints for drug development in advanced melanoma. Clin. Cancer Res. 18, 336-341 (2012).

10 Georgetown Lombardi Comprehensive Cancer Center, Schafer Memorial Lecture. https://ruesch. georgetown.edu/about/symposium/schafer_lecture

11 Winslow R. Cancer's super-survivors - how the promise of immunotherapy is transforming oncology. Wall Street Journal 4 December 2014.

12 Couzin-Frankel J. Cancer immunotherapy. Science 342, 1432-1433 (2013).

13 Onclive. New paradigms emerge for translating immunotherapy into broad clinical use. www.onclive. com/publications/Oncology-live/2014/February2014/New-Paradigms-Emerge-for-TranslatingImmunotherapy-Into-Broad-Clinical-Use

14 Alexandrov LB, Nik-Zainal S, Wedge DC et al. Signatures of mutational processes in human cancer. Nature 500, 415-421 (2013).
15 Herbst RS, Soria JC, Kowanetz M et al. Predictive correlates of response to the anti-PD-L1 antibody MPDL3280A in cancer patients. Nature 515, 563-567 (2014).

16 Hilton T, Hulett T, Dubay C et al. DPV-001 an autophagosome-enriched cancer vaccine in Phase II clinical trials contains 25 putative cancer antigens, DAMPS, HSPS and agonists for TLR 2, 3, 4, 7 and 9. J. Immunother. Cancer 1, P260 (2013).

17 Schlom J. Therapeutic cancer vaccines. Adv. Cancer Res. 121, 67-124 (2014).

18 Center For Cancer Research, Jeffrey Schlom. https://ccr.cancer.gov/jeffrey-schlom

19 Hamilton DH, Litzinger MT, Jales A et al. Immunological targeting of tumor cells undergoing an epithelial-mesenchymal transition via a recombinant brachyury-yeast vaccine. Oncotarget 4, 1777-1790 (2013).

20 Le DT, Wang-Gillam A, Picozzi V et al. Safety and survival with GVAX pancreas prime and Listeria monocytogenes-expressing mesothelin (CRS-207) boost vaccines for metastatic pancreatic cancer. J. Clin. Oncol. pii:JCO.2014.57.4244 (2015) (Epub ahead of print).

21 Wolchok JD, Kluger H, Callahan MK et al. Nivolumab plus ipilimumab in advanced melanoma. N. Engl. J. Med. 369, 122-133 (2013). 